\title{
Archivos de Cardiología de México

\section{Síndrome de Andersen-Tawil: una revisión del diagnóstico genético y clínico con énfasis en sus manifestaciones cardíacas}

\author{
Manlio F. Márquez ${ }^{\mathrm{a}, *}$, Armando Totomoch-Serra ${ }^{\mathrm{b}, \mathrm{c}}$, Gilberto Vargas-Alarcón ${ }^{\mathrm{b}}$, \\ David Cruz-Robles ${ }^{\mathrm{b}}$, Oscar A. Pellizzon ${ }^{\mathrm{d}}$ y Manuel Cárdenas ${ }^{\mathrm{a}}$ \\ a Departamento de Electrofisiología, Instituto Nacional de Cardiología Ignacio Chávez, México D.F., México \\ b Departamento de Biología Molecular, Instituto Nacional de Cardiología Ignacio Chávez, México D.F., México \\ c Maestría en Investigación Clínica Experimental en Salud, Universidad Nacional Autónoma de México, México D.F., México \\ d Centro de Arritmias Cardíacas, Hospital Universitario del Centenario, Rosario (Santa Fe), Argentina
}

Recibido el 5 de diciembre de 2013; aceptado el 12 de diciembre de 2013

\section{PALABRAS CLAVE \\ Síndrome de Andersen-Tawil; \\ Síndrome de QT \\ largo; \\ Mutación génica; \\ Diagnóstico; \\ Clasificación; \\ México}

\begin{abstract}
Resumen El síndrome de Andersen-Tawil resulta de la alteración de canales de potasio, se hereda de forma autosómica dominante y se cataloga como el tipo 7 de los síndromes de QT largo congénitos. El gen afectado es el KCNJ2, el cual codifica la proteína Kir2.1 que forma el canal de potasio rectificador interno ( inward rectifier»). Este canal interviene en la estabilización del potencial de membrana en reposo y controla la duración del potencial de acción en el sistema musculoesquelético y cardíaco. En miocitos ventriculares, es un componente responsable de la rectificación de la corriente de potasio en la fase 3 del potencial de acción. Debido a que Kir2.1 está presente en el sistema musculoesquelético, corazón y cerebro, las alteraciones de esta proteína dan origen a las principales características del síndrome: parálisis flácida, arritmias ventriculares y alteraciones leves a moderadas en el desarrollo del esqueleto, especialmente en manos y pies. En la presente revisión se aborda esta enfermedad desde el punto de vista del diagnóstico clínico y molecular con énfasis en sus manifestaciones cardíacas.

(C) 2013 Instituto Nacional de Cardiología Ignacio Chávez. Publicado por Masson Doyma México S.A. Todos los derechos reservados.
\end{abstract}

Andersen-Tawil syndrome: A review of its clinical and genetic diagnosis with emphasis on cardiac manifestations

Abstract The Andersen-Tawil syndrome is a cardiac ion channel disease that is inherited in an autosomal dominant way and is classified as type 7 of the congenital long QT syndromes. Affected gene is $K C N J 2$, which forms the inward rectifier potassium channel designated Kir2.1. This protein is involved in stabilizing the resting membrane potential

\section{KEYWORDS}

Andersen-Tawil syndrome; Long QT syndrome;

Gene mutation;

Diagnosis;

Classification; Mexico

\footnotetext{
* Autor para correspondencia.

Correo electrónico: manlio.marquez@gmail.com (M.F. Márquez).
} 
and controls the duration of the action potential in skeletal muscle and heart. It also participates in the terminal repolarization phase of the action potential in ventricular myocytes and is a major component responsible for the correction in the potassium current during phase 3 of the action potential repolarization. Kir 2.1 channel has a predominant role in skeletal muscle, heart and brain. Alterations in this channel produce flaccid paralysis, arrhythmias, impaired skeletal development primarily in extremities and facial area. In this review we address the disease from the point of view of clinical and molecular diagnosis with emphasis on cardiac manifestations.

( 2013 Instituto Nacional de Cardiología Ignacio Chávez. Published by Masson Doyma México S.A. All rights reserved.

\section{Introducción}

El síndrome de Andersen-Tawil (ATS, por sus siglas en inglés) es una enfermedad de canales iónicos donde el gen afectado, el KCNJ2 (locus 17q23-q24.2), codifica el canal de potasio rectificador interno Kir2. $1^{1}$. Se cataloga como el tipo 7 de los síndromes de QT largo (SQTL) congénito², aunque existe cierta controversia al respecto ${ }^{3}$. En esta revisión se aborda a la enfermedad desde el punto de vista del diagnóstico clínico y molecular con énfasis en sus manifestaciones cardíacas.

\section{Antecedentes}

El primer caso clínico de esta enfermedad fue informado por Ellen Andersen, Peter Krasilnikoff y Hans Overvad del Hospital Universitario de Copenhague (Dinamarca), en la revista Acta Pediatrica Scandinavica (1971) y correspondió a un niño de 8 años de edad con 3 características clínicas principales: debilidad muscular (parálisis periódica) intermitente, arritmias (extrasístoles ventriculares) y alteraciones en el desarrollo (talla baja, cabello delgado, dolicocefalia, puente nasal ancho, implantación baja de pabellones auriculares, paladar hendido, dentición retrasada e incompleta, hipoplasia mandibular, pliegue palmar transverso, clinodactilia del quinto dedo de las manos y pies, así como mineralización incompleta del cráneo) ${ }^{4}$. Tawil et al. ${ }^{5}$ informaron de 4 pacientes con fenotipo similar e hicieron una mejor descripción del espectro clínico de la enfermedad. Fueron los primeros en utilizar el epónimo síndrome de Andersen. Aunque se señaló en su momento que probablemente el nombre correcto debía ser síndrome de Klein-Lisak-Andersen, debido a la existencia de publicaciones anteriores a Andersen por parte de los 2 primeros autores $^{6}$, este epónimo no prosperó y tiempo después pasó a denominarse síndrome de Andersen-Tawil debido a que el Dr. Tawil desarrolló los criterios diagnósticos y demostró que este síndrome es distinto de otras formas de parálisis periódica sensible al potasio por medio de un «estudio de ligamiento», señalando incluso la posibilidad de que se tratase de una forma distinta de SQTL ${ }^{7}$. En 1997, Sansone et al. enfatizaron la presencia de la tríada clásica en 11 pacientes $^{7}$. El primer informe en México del síndrome fue de Canún, Pérez y Beirana ${ }^{8}$ y consistió en una descripción clínica muy completa en 3 generaciones de una misma familia.
La muestra sanguínea del caso índice y sus familiares fue enviada a EE. UU. donde, junto con pacientes de otras latitudes, se describió por primera vez la mutación causante del síndrome en el gen $\mathrm{KCNJ}^{9}$.

\section{Bases moleculares de la enfermedad}

Mediante un estudio de «análisis de ligamiento» con 400 marcadores polimórficos en el genoma de 15 individuos con ATS, Plaster et al. ${ }^{9}$ identificaron que la región candidata se localizaba en el locus 17q23 por medio del marcador D17S949 ${ }^{10}$, obteniendo un lod $=3.23$ y theta $=0.0$. Como en este locus se encuentra un gen que codifica para un canal de potasio, el $K C N J 2$, decidieron estudiarlo, identificando la mutación D71V en la familia mexicana informada previamente por Canún et al. ${ }^{8}$. Esta mutación tiene un efecto dominante negativo y corresponde a una transversión (cambio de adenina por timina) en el nucleótido 440 que resulta en un cambio en el dominio aminoterminal en la última posición de una alfa-hélice; este residuo es apenas distal al dominio putativo de interacción $\mathrm{N}$-terminal ${ }^{11,12}$

Actualmente se conoce que el gen $K C N J 2$ se localiza en la región 17q23.1-q24.2, tiene una longitud de 11,372 pares de bases, su marco de lectura no se encuentra interrumpido por intrones, tiene 2 exones y 2 transcritos, uno de 5,392 y otro de 1,685 pares de bases. Codifica para una proteína que forma el canal rectificador interno de potasio llamado Kir2.1. Este canal, formado por 427 aminoácidos, tiene un peso molecular de $48 \mathrm{kD}$ y 2 regiones membranales conectadas por un dominio en forma de poro y dominios $\mathrm{N}$ y C-terminales citoplasmáticos ${ }^{13,14}$ (fig. 1).

Kir2.1 es un canal rectificador interno «fuerte», contribuye a la corriente IK1 que interviene en la estabilización del potencial de membrana en reposo y controla la duración del potencial de acción en el sistema musculoesquelético y cardíaco; tiene un papel en la fase de repolarización terminal del potencial de acción. En miocitos ventriculares es un componente mayor responsable de la rectificación en la corriente de potasio en la fase 3 del potencial de acción ${ }^{15,16}$. La actividad eléctrica espontánea del ventrículo, secundaria a supresión de la corriente de potasio, puede explicar la mayor frecuencia de ectopia ventricular en el ATS a diferencia de otros tipos de SQTL. La pérdida de función de Kir2.1, secundaria a diferentes mutaciones, prolonga el potencial de acción ventricular lo que se 
traduce en un alargamiento del intervalo QT-U y favorece la aparición de taquiarritmias ventriculares. Las subunidades alfa de los canales Kir están formadas por 2 segmentos transmembranales y un poro. Debido a que estas 4 subunidades alfa están ensambladas para funcionar como un tetrámero, se considera que una mutación en una sola región crítica puede ser suficiente para dañar la función del canal completo ${ }^{11}$. A la fecha, 2 sitios de mutación reconocidos se encuentran en los residuos de unión a fosfatidilinositol-45-bifosfato localizado en el extremo C-terminal y en el asa («loop») del poro o la región externa de Kir2.1. Estas mutaciones causan modificación de la hélice del poro que alteran la selectividad y conformación del filtro o interfieren con el desempeño de la hélice al disminuir la barrera electrostática para la conducción iónica ${ }^{15-18}$.

Las deleciones de los residuos 95-98 remueven una región del primer dominio transmembranal de Kir2.1. La alteración de estos 4 aminoácidos cambia la formación de la alfa-hélice en la membrana y consecuentemente la inserción del canal en la membrana; adicionalmente no se puede mantener un estado abierto o incluso manejar los iones de potasio ${ }^{19}$. Andelfinger et al. ${ }^{20}$ identificaron una mutación de cambio de sentido, arginina 67 por triptófano (R67W), en 41 miembros de una familia en la cual no todos los individuos tenían manifestaciones de ATS, lo que sugiere penetrancia incompleta. En una familia con la mutación R67W en KCNJ2 se observó parálisis periódica solo en hombres, síntomas cardíacos en mujeres y anomalías congénitas en ambos. Este tipo de segregación, limitada al sexo, no ha sido confirmada. Aunque en la actualidad no existe dentro del gen KNCJ2 una mutación predominante o un sitio que concentre la mayoría de las mutaciones («sitio caliente» o «hot-spot» en inglés), cada vez se informan más pacientes con ATS con la mutación R67W, por lo que el sitio donde se localiza esta mutación se ha llegado a considerar como un potencial «hot-spot» ${ }^{21,22}$.

Hasta la fecha se han descrito 62 mutaciones en el gen KCNJ2 para ATS, la mayoría se relacionan con un efecto dominante negativo (cuando interaccionan 2 o más proteínas, pero una de ellas es anómala [desestabilizando de esta forma el tetrámero]) aunque también hay casos de haploinsuficiencia (cuando disminuye la cantidad de proteína necesaria para formar el canal) ${ }^{11,19-22}$. También se ha encontrado pérdida de la función del canal debida a una alteración que impide el transporte de las proteínas del canal desde el retículo endoplásmico hacia la membrana ${ }^{23}$. En la figura 1 se presenta un esquema de la localización de las 62 mutaciones descritas hasta el momento en el canal Kir2.1. Se puede apreciar que la mayoría ocurren en ambas «colas citoplasmáticas», predominantemente en el extremo carboxiterminal.

\section{Fisiopatología}

Kir2.1 tiene una función predominante en el sistema musculoesquelético, corazón y cerebro, por lo que la alteración de este canal en el ATS conlleva: parálisis flácida, arritmias ventriculares y alteraciones leves a moderadas en el desarrollo del esqueleto ${ }^{24-28}$. La parálisis flácida por la incapacidad para propagar el potencial de acción en la membrana muscular es resultado de la despolarización sostenida de la membrana celular ${ }^{26,27}$. La prolongación del intervalo QT-U y las arritmias ventriculares son causadas por la alteración en la repolarización ventricular ${ }^{25,28}$. Aunque el papel de Kir2.1

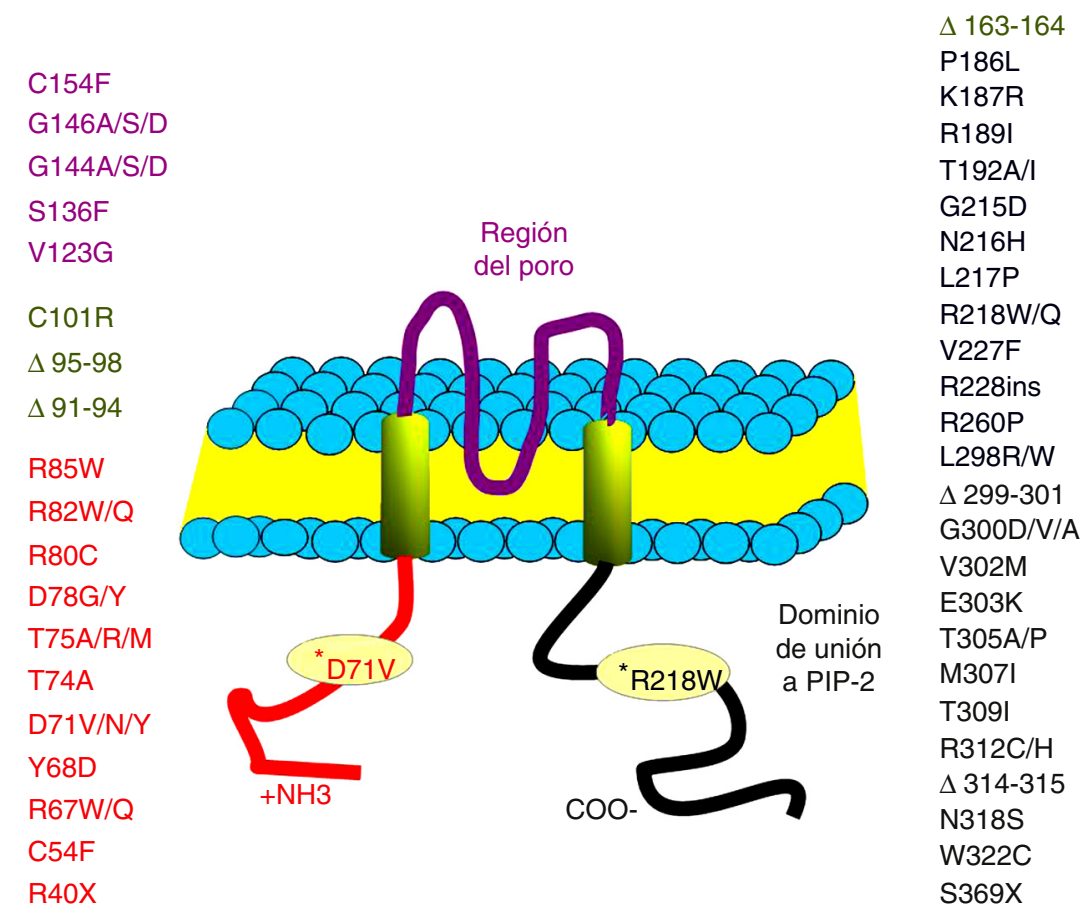

Figura 1 Mutaciones en el canal Kir2.1. Se puede apreciar que la mayoría ocurren en el dominio C-terminal. Con asterisco, las mutaciones descritas en familias mexicanas. La D71V identificada por Plaster et al. ${ }^{9}$ y corroborada en el INCICh en los demás miembros de la familia y la R218W identificada por Sistemas Genómicos ${ }^{\circledR}$ S.L. (Valencia España). 
en las alteraciones del desarrollo esquelético está por ser aclarado, la corriente de potasio en Kir2.1 es necesaria para la función de los osteoblastos. Se sabe que el intercambio de iones potasio por hidrógeno es necesario para mantener el medio ácido adecuado para una reabsorción ósea normal. Estudios realizados en ratones modificados por ingeniería genética para que uno o más de sus genes estén inactivos muestran letalidad perinatal por deshidratación o insuficiencia respiratoria secundarias a paladar hendido, anomalía que ocurre en el $100 \%$ de ellos ${ }^{29}$. Se desconoce la razón por la cual este modelo no experimenta arritmias ni parálisis periódica.

Para explicar la conocida variabilidad en la expresión fenotípica del ATS se han postulado varias hipótesis. Una de ellas la considera consecuencia de heteromultimerización (unión de subunidades de diferentes proteínas) de Kir2.1 con homólogos de la familia Kir2.x, específicamente Kir2.2 y Kir2.3 $3^{30}$. Otro gen que interactúa con Kir2.1 es el de la anquirina $B$, proteína que funciona como un adaptador proteico que está relacionada con el ensamble de la ATPasa de sodio/potasio. Algunas otras proteínas que interactúan con Kir2.1 son la proteína cinasa de anclaje 79, la proteína asociada a sinapsis 97 , el complejo de proteína de densidad postsináptica 95 / proteína sináptica asociada y la filamina $\mathrm{A}^{19}$.

\section{Características clínicas}

Uno de los primeros síntomas es la debilidad muscular después de una actividad física prolongada; esta comienza en la infancia o adolescencia, puede remitir espontáneamente y su duración va desde unas horas a días. La prueba neurofisiológica (electromiografía pre- y postejercicio) de McManis modificada por Fournier es de utilidad para la detección de ATS en pacientes con parálisis periódica ${ }^{26,27}$. Las crisis de parálisis periódica no parecen depender de la concentración de potasio, que puede estar alta, normal o disminuida por lo que idealmente la medición de este electrólito debe realizarse durante dichos episodios. En el subgrupo de pacientes donde hay hipocaliemia hay una respuesta satisfactoria a la administración de potasio ${ }^{24}$. En la tabla 1 se mencionan los criterios diagnósticos para ATS.

Desde el punto de vista del diagnóstico molecular, como hasta el día de hoy no se han descrito mutaciones en otro gen distinto al $K C N J 2$, los individuos con ATS se clasifican como ATS1 si se encuentra alguna mutación en KCNJ2 y como ATS2 si se estudia este gen y no se encuentra ninguna mutación. Es importante aclarar que desde el punto de vista fenotípico, ATS1 y ATS2 son indistinguibles.

Aunque las extrasístoles ventriculares y la taquicardia ventricular no sostenida son muy frecuentes, es menos común la presencia de síncope arrítmico, paro cardíaco o muerte súbita ${ }^{31,32}$. Esta discordancia entre una carga arrítmica elevada (extrasístoles y taquicardias ventriculares frecuentes) y la aparente baja incidencia de muerte súbita es un asunto que no ha sido explorado en profundidad. En la tabla 2 se mencionan las características ecocardiográficas y electrocardiográficas que se han señalado en el ATS. Aunque la mayoría de los casos se presentan en corazón estructuralmente sano, en algunos se ha observado la presencia de miocardiopatía dilatada ${ }^{33}$. No se ha definido si esta es una manifestación poco común de la enfermedad o una consecuencia de la taquicardia crónica («taquicardiomiopatía»). Se ha documentado y publicado un caso en el cual la eliminación de la taquicardia ventricular, mediante el uso de flecainida, ha logrado revertir la dilatación ventricular ${ }^{34}$. Otras características descritas, pero menos comunes en ATS, son las malformaciones cardiovasculares, especialmente de los aparatos valvulares ${ }^{20}$.

Un dato común en los pacientes con ATS es la presencia de un intervalo QT-U largo (fig. 2), con un patrón característico

Tabla 1 Criterios diagnósticos de síndrome de Andersen-Tawil

Clínicamente sospecha de ATS en individuos con características de la sección A o B:
$\begin{array}{ll}\text { Sección A } & \text { I. Parálisis periódica }\end{array}$

Dos de 3 criterios

II. Arritmia sintomática o evidencia electrocardiográfica de ectopia ventricular, aumento en el voltaje de la onda $U$ o de alargamiento del intervalo QTC o Q-Uc

III. Facies característica (frente amplia, fisuras palpebrales cortas, puente nasal amplio, punta de la nariz bulbosa, hipoplasia mandibular y maxilar, labio superior delgado y cara triangular) alteraciones dentales, manos y pies pequeños, y al menos 2 de los siguientes datos:

1. Implantación baja de pabellones auriculares

2. Hipertelorismo

3. Micrognatia

4. Clinodactilia del quinto dedo

5. Sindactilia

Sección B Uno de los 3 criterios arriba mencionados, más lo siguiente: Que exista en la familia por lo menos un integrante diagnosticado con 2 de los 3 criterios de la sección A 
Tabla 2 Alteraciones eléctricas y ecocardiográficas descritas en el síndrome de Andersen-Tawil

\begin{tabular}{ll}
\hline Datos electrocardiográficos & Datos ecocardiográficos \\
\hline Onda U prominente o bifásica & Malformaciones valvulares \\
Intervalo QTc/Q-Uc prolongado & Estenosis de la pulmonar \\
Bigeminismo ventricular & Válvula aórtica bicúspide, con y sin coartación \\
Extrasístoles ventriculares & \\
Taquicardia ventricular & \\
Bidireccional & \\
Polimórfica & \\
Helicoidal & \\
Bloqueo auriculoventricular & \\
Bloqueo de rama (derecha o izquierda) & \\
Bloqueo bifascicular &
\end{tabular}

T-U el cual incluye onda $U$ prolongada, intervalo T-U amplio y onda $\mathrm{T}$ terminal prolongada con un declive característico para ATS1 en comparación con los otros SQTL ${ }^{28}$.

\section{Asesoramiento genético}

Como ya se ha señalado, el ATS se hereda de forma autosómica dominante. Al menos el $50 \%$ de los individuos diagnosticados con ATS tienen un padre afectado, más del $50 \%$ son causados por una mutación de novo. Cada hijo de un individuo con ATS tiene un 50\% de probabilidad de heredar la enfermedad (fig. 3); un factor que puede disminuir esta probabilidad es la falta de penetrancia, la cual es evidente en el $6-20 \%$ de los individuos con una mutación identificada. Para llevar a cabo el diagnóstico prenatal en una gestante en quien ya se haya identificado la mutación, en ella o en su familia, se puede realizar el análisis genético del producto mediante amniocentesis o biopsia de vellosidades coriales $^{35,36}$. Hasta la fecha no hay informes de diagnóstico pre-implantacional que podría ser una opción en familias en las que se conoce la mutación.

\section{Tratamiento antiarrítmico}

El tratamiento antiarrítmico es controvertido debido a la baja prevalencia de la enfermedad y a la variabilidad en la expresión del fenotipo. Existen pocas publicaciones al respecto y la mayoría son informes de casos aislados o pequeñas series; el objetivo en ellas ha variado, desde disminuir la ectopia ventricular, eliminar la taquicardia ventricular, hasta prevenir la muerte súbita.

En la tabla 3 se presenta un resumen de la literatura. Para la mayoría de los fármacos, los resultados son contradictorios, con informes aislados de su utilidad e informes de su ineficacia ${ }^{37-42}$. El medicamento que parece ser más útil es la

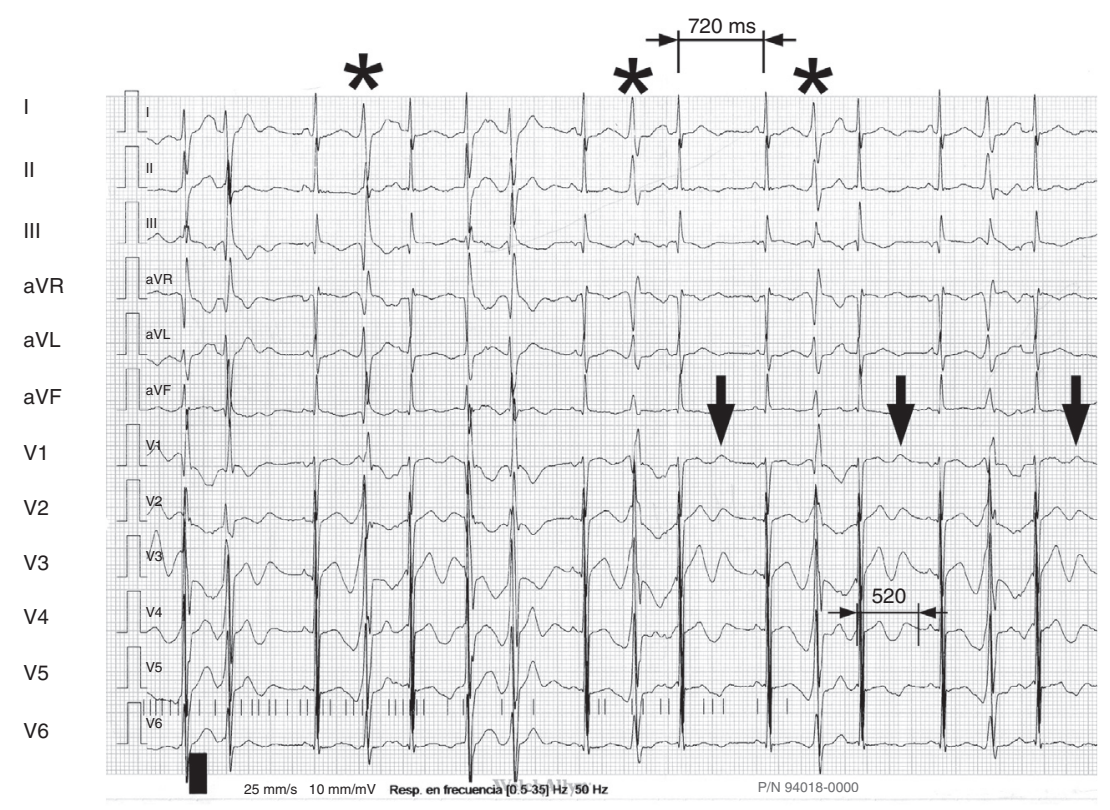

Figura 2 Electrocardiograma de 12 derivaciones en donde se observa ritmo sinusal interrumpido por extrasístoles ventriculares monomórficas (asteriscos) que salen de la rama ascendente de la onda U. Nótese la prolongación del intervalo QT-U (520 ms) a expensas de una onda $U$ (flechas) de gran amplitud y magnitud (véanse las derivaciones V1 a V4). QU corregido (RR 720 ms) = 612. 


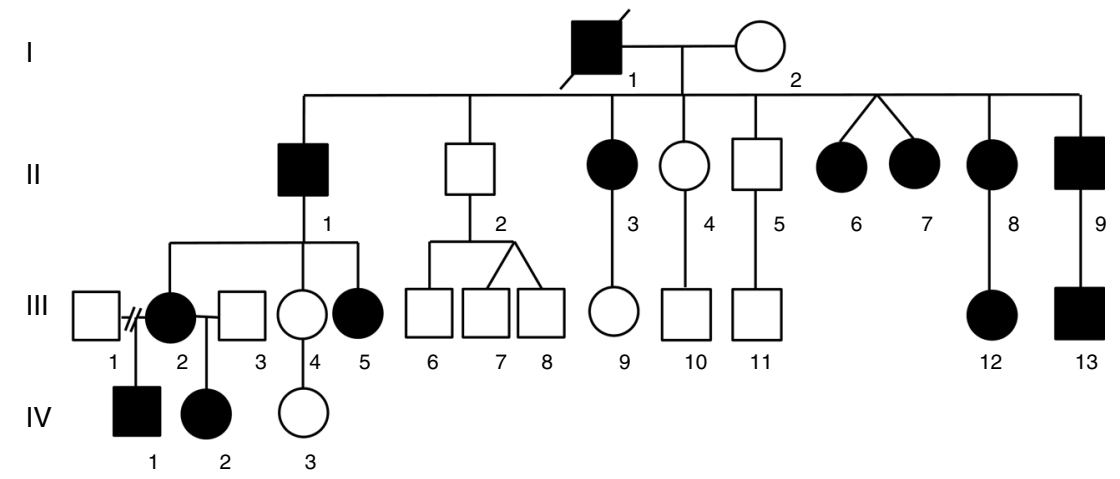

Figura 3 Árbol genealógico actualizado de la familia mexicana con ATS descrita originalmente por Canún et al. ${ }^{8}$. Por el tiempo transcurrido, se ha agregado una cuarta generación cuyos miembros tienen el patrón de herencia autosómico dominante.

flecainida debido a que hay más publicaciones a favor que en contra de su uso. En casos seleccionados, la flecainida fue muy eficaz para eliminar extrasístoles ventriculares, TV bidireccional e incluso taquicardiomiopatía ${ }^{34,43,44}$. También se han empleado los betabloqueadores, ya sea solos en combinación con flecainida ${ }^{32}$ y los calcioantagonistas ${ }^{45}$ (tabla 3 ). De cualquier manera, es importante señalar que en la serie de Delannoy et al. ${ }^{32}$ hubo un caso de paro cardíaco no fatal

Tabla 3 Fármacos empleados para el tratamiento antiarrítmico en el síndrome de Andersen-Tawil. Se distinguen entre aquellos informados como eficaces o ineficaces

\begin{tabular}{|c|c|c|c|c|c|}
\hline Autores & Sexo & Edad & DAI & Informados eficaces & Ineficaces \\
\hline Obeyesekere et al. ${ }^{37}$ & $M$ & 53 & Sí & Betabloqueadores & $\mathrm{NI}$ \\
\hline Barajas-Martinez et al. ${ }^{21}$ & $\mathrm{~F}$ & 10 & No & Flecainida & Nadolol \\
\hline \multirow[t]{2}{*}{ Schoonderwoerd et al. ${ }^{33}$} & $\mathrm{~F}$ & 18 & Sí & Ninguno & Metoprolol \\
\hline & M & 16 & No & Metoprolol (oral) & $\mathrm{NI}$ \\
\hline Weir et al. ${ }^{38}$ & $\mathrm{~F}$ & 34 & No & Atenolol & $\begin{array}{l}\text { Propranolol Disopiramida, } \\
\text { amiodarona, flecainida, } \\
\text { imipramina }\end{array}$ \\
\hline Pellizzón et al. ${ }^{34}$ & $\mathrm{~F}$ & 16 & No & Flecainida & $\begin{array}{l}\text { Lidocaína, propranolol, } \\
\text { diltiazem, potasio, sulfato } \\
\text { de magnesio (todos iv) }\end{array}$ \\
\hline Subbiah et al. ${ }^{35}$ & $\mathrm{~F}$ & 27 & No & Metoprolol (iv) & $\mathrm{NI}$ \\
\hline Sansone y Tawil ${ }^{39}$ & $\mathrm{NI}$ & $\mathrm{NI}$ & No & $\begin{array}{l}\text { Propranolol amlodipino, } \\
\text { nifedipino }\end{array}$ & $\mathrm{NI}$ \\
\hline \multirow[t]{2}{*}{ Haruna et al. ${ }^{40}$} & $M(n=3)$ & $11^{\mathrm{a}}$ & Sí & Propranolol, atenolol, & $\mathrm{NI}$ \\
\hline & $F(n=7)$ & $21^{a}$ & $(n=1)$ & $\begin{array}{l}\text { metoprolol o carvedilol } \\
(n=7) \text {, verapamilo }(5), \\
\text { flecainida }(n=1) \\
\text { mexiletina }(n=2)\end{array}$ & \\
\hline \multirow[t]{2}{*}{ Bökenkamp et al. ${ }^{43}$} & $\mathrm{~F}$ & 7 & Sí & $\begin{array}{l}\text { Flecainida(la niña tuvo } \\
\text { una descarga apropiada } \\
\text { bajo tratamiento) }\end{array}$ & Propranolol, verapamilo \\
\hline & M & 6 & No & Flecainida & $\mathrm{NI}$ \\
\hline Fox et al. ${ }^{44}$ & M & 54 & No & Flecainida & Bisoprolol \\
\hline Junker et al. ${ }^{41}$ & $\mathrm{~F}$ & 18 & Sí & Amiodarona & $\begin{array}{l}\text { Sotalol, flecainida, } \\
\text { propafenona }\end{array}$ \\
\hline Garcia-Touchard et al. ${ }^{42}$ & $\mathrm{~F}$ & 16 & No & Mexiletina + atenolol & Atenolol \\
\hline Erdogan et al. ${ }^{45}$ & $\mathrm{~F}$ & 65 & No & Verapamilo & $\begin{array}{l}\text { Betabloqueador } \\
\text { Propafenona } \\
\text { Sotalol }\end{array}$ \\
\hline Delannoy et al. ${ }^{32}$ & $28 \mathrm{pts}$ & $\mathrm{NI}$ & 3 & $\begin{array}{l}\text { Betabloqueador } \\
\text { - Solo }(n=20) \\
\text { - Con flecainida }(n=6) \\
\text { - Con amiodarona }(n=2)\end{array}$ & $\begin{array}{l}\text { Betabloqueador con } \\
\text { flecainida (un paciente } \\
\text { presentó paro cardíaco } \\
\text { con este tratamiento) }\end{array}$ \\
\hline
\end{tabular}

DAl: desfibrilador automático implantable; Iv: intravenoso; NI: no informado.

a Edad promedio. 
(gracias a que tenía un cardiodesfibrilador) a pesar del tratamiento con betabloqueador y flecainida.

Se ha informado de la utilidad de la ablación por radiofrecuencia para el tratamiento de arritmias ventriculares malignas en otros síndromes genéticos como el de Brugada ${ }^{46}$ por lo que este tratamiento podría ser atractivo en el ATS debido a la frecuente ectopia ventricular. Sin embargo, hasta la fecha no se ha realizado ningún informe sobre ablación exitosa en el ATS ${ }^{3}$. En una serie de 33 casos con ATS, se informó que se intentó la ablación en 5 casos y en ninguno tuvo éxito ${ }^{32}$. En una paciente de la serie de ATS del Instituto Nacional de Cardiología se intentó la ablación con catéter en forma infructuosa en 2 ocasiones (datos no publicados).

Al igual que en otros síndromes de muerte súbita, el cardiodesfibrilador automático implantable es una indicación de clase I en aquellos pacientes con paro cardíaco por fibrilación ventricular, síncopes recurrentes o taquicardia ventricular sintomática ${ }^{47}$.

\section{Conclusión}

El ATS tiene un fenotipo característico por lo que es indispensable que los médicos, en particular los cardiólogos, lo puedan identificar así como que conozcan su importancia desde el punto de vista de su asociación con muerte súbita para poder asesorar a los pacientes y a sus familiares.

\section{Financiación}

Ninguno.

\section{Conflicto de intereses}

Los autores declaran no tener ningún conflicto de intereses.

\section{Agradecimientos}

A la compañía Sistemas Genómicos $®$ S.L. (Valencia, España) por la secuenciación masiva (next generation sequencing) para la determinación de la mutación KCNJ2 (R218W) de la segunda familia mexicana con ATS reflejada en la figura 1.

\section{Bibliografía}

1. Abriel H, Zaklyazminskaya EV. Cardiac channelopathies: Genetic and molecular mechanisms. Gene. 2013;517:1-11.

2. Tristani-Firouzi M, Jensen JL, Donaldson MR, et al. Functional and clinical characterization of KCNJ2 mutations associated with LQT7 (Andersen syndrome). J Clin Invest. 2002;110:381-8.

3. Wilde AA. Andersen-Tawil syndrome, scarier for the doctor than for the patient? Who, when, and how to treat. Europace. 2013; 15:1690-2.

4. Andersen ED, Krasilnikoff PA, Overvad H. Intermittent muscular weakness, extrasystoles and multiple developmental abnormalities: A new syndrome. Acta Paediatr Scand. 1971;60:559-64.

5. Tawil R, Ptácek LJ, Pavlakis SG, et al. Andersen's syndrome: Potassium-sensitive periodic paralysis, ventricular ectopy, and dysmorphic features. Ann Neurol. 1994;35:326-30.

6. Rowland LP. Andersen's syndrome? Or Hein-Lisak-Andersen syndrome? Ann Neurol. 1994;35:252-3.
7. Sansone V, Griggs RC, Meola TG, et al. Andersen's syndrome: A distinct periodic paralysis. Ann Neurol. 1997;42:305-12.

8. Canún S, Pérez N, Beirana LG. Andersen syndrome autosomal dominant in three generations. Am J Med Genet. 1999;85:147-56.

9. Plaster NM, Tawil R, Tristani-Firouzi M, et al. Mutations in Kir2.1 cause the developmental and episodic electrical phenotypes of Andersen's syndrome. Cell. 2001;105:511-9.

10. Genbank 2014 [consultado 1 Ene 2014]. Disponible en: http://www.ncbi.nlm.nih.gov/nuccore/Z24111.1

11. Lu CW, Lin JH, Rajawat YS, et al. Functional and clinical characterization of a mutation in KCNJ2 associated with Andersen-Tawil syndrome. J Med Genet. 2006;43:653-9.

12. Marrus SB, Cuculich PS, Wang W, et al. Characterization of a novel, dominant negative KCNJ2 mutation associated with Andersen-Tawil syndrome. Channels. 2011;5:500-9.

13. Tristani-Firouzi M, Etheridge SP. Kir2.1 channelopathies: The Andersen-Tawil syndrome. Pflugers Arch. 2010;460: 289-94.

14. Lim BC, Kim GB, Bae EJ, et al. Andersen cardiodysrhythmic periodic paralysis with $K C N J 2$ mutations: A novel mutation in the pore selectivity filter residue. J Child Neurol. 2010;25:490-3.

15. Tsuboi M, Antzelevitch C. Cellular basis for electrocardiographic and arrhythmic manifestations of Andersen-Tawil syndrome (LQT7). Heart Rhythm. 2006;3:328-35.

16. Gómez Garcia R. Regulación de los canales kir2. 1 y de la corriente cardíaca humana $I_{k 1}$ por el óxido nítrico [tesis doctoral]. Madrid: Facultad de Medicina, Departamento de Farmacología, Universidad Complutense de Madrid; 2010.

17. Ma D, Tang XD, Rogers TB, et al. An Andersen-Tawil syndrome mutation in Kir2.1 (V302M) alters the G-loop cytoplasmic K+ conduction pathway. J Biol Chem. 2007;282:5781-9.

18. Xiao J, Zhen XG, Yang J. Localization of PIP2 activation gate in inward rectifier $\mathrm{K}+$ channels. Nat Neurosci. 2003;6: 811-8.

19. Bendahhou S, Donaldson MR, Plaster NM, et al. Defective potassium channel Kir2.1 trafficking underlies Andersen-Tawil syndrome. J Biol Chem. 2003;278:51779-85.

20. Andelfinger G, Tapper AR, Welch RC, et al. KCJN2 mutation results in Andersen syndrome with sex-specific cardiac and skeletal muscle phenotypes. Am J Hum Genet. 2002;71: 663-8.

21. Barajas-Martinez H, Hu D, Ontiveros G, et al. Biophysical and molecular characterization of a novel de novo KCNJ2 mutation associated with Andersen-Tawil Syndrome and catecholaminergic polymorphic ventricular tachycardia mimicry. Circ Cardiovasc Genet. 2011;4:51-7.

22. Kimura H, Zhou J, Kawamura M, et al. Phenotype variability in patients carrying KCNJ2 mutations. Circ Cardiovasc Genet. 2012;5:344-53.

23. Doi T, Makiyama T, Morimoto $T$, et al. A novel KCNJ2 nonsense mutation, S369X, impedes trafficking and causes a limited form of Andersen-Tawil syndrome. Circ Cardiovasc Genet. 2011;4:253-60.

24. Jurkat-Rott K, Lehmann-Horn F. Genotype-phenotype correlation and therapeutic rationale in hyperkalemic periodic paralysis. Neurotherapeutics. 2007;4:216-24.

25. Sung RJ, Wu SN, Wu JS, et al. Electrophysiological mechanisms of ventricular arrhythmias in relation to Andersen-Tawil syndrome under conditions of reduced $\mathrm{I}_{\mathrm{k} 1 \text { : }}$ a simulation study. Am J Physiol Heart Circ Physiol. 2006;291:H2597-605.

26. Fournier E, Arzel M, Sternberg D, et al. Electromyography guides toward subgroups of mutations in muscle channelopathies. Ann Neurol. 2004;56:650-1.

27. Diaz-Manera J, Querol L, Clarimón J, et al. Unique post-exercise electrophysiological test results in a new Andersen-Tawil syndrome mutation. Clin Neurophysiol. 2011;122:2537-9. 
28. Zhang L, Benson DW, Tristani-Firouzi M, et al. Electrocardiographic features in Andersen-Tawil syndrome patients with KCNJ2 mutations: Characteristic T-U-wave patterns predict the KCNJ2 genotype. Circulation. 2005;111:2720-6.

29. Zaritsky JJ, Eckman DM, Wellman GC, et al. Targeted disruption of Kir2.1 and Kir2.2 genes reveals the essential role of the inwardly rectifying $\mathrm{K}(+)$ current in $\mathrm{K}(+)$-mediated vasodilation. Circ Res. 2000;87:160-6.

30. Preisig-Müller R, Schlichthörl G, Goerge T, et al. Heteromerization of Kir2.x potassium channels contributes to the phenotype of Andersen's syndrome. Proc Natl Acad Sci U S A. 2002;99:7774-9.

31. Peters S, Schulze-Bahr E, Etheridge SP, et al. Sudden cardiac death in Andersen-Tawil syndrome. Europace. 2007;9:162-6.

32. Delannoy E, Sacher F, Maury P, et al. Cardiac characteristics and long-term outcome in Andersen-Tawil syndrome patients related to KCNJ2 mutation. Europace. 2013;15:1805-11.

33. Schoonderwoerd BA, Wiesfeld AC, Wilde AA, et al. A family with Andersen-Tawil syndrome and dilated cardiomyopathy. Heart Rhythm. 2006;3:1346-50.

34. Pellizzón OA, Kalaizich L, Ptacek LJ, et al. Flecainide suppresses bidirectional ventricular tachycardia and reverses tachycardia-induced cardiomyopathy in Andersen-Tawil syndrome. J Cardiovasc Electrophysiol. 2008;19:95-7.

35. Subbiah RN, Gula LJ, Skanes AC, et al. Andersen-Tawil syndrome: Management challenges during pregnancy, labor, and delivery. J Cardiovasc Electrophysiol. 2008;19:987-9.

36. Ingles J, Yeates $L$, Semsarian $C$. The emerging role of the cardiac genetic counselor. Heart Rhythm. 2011;8:1958-62.

37. Obeyesekere MN, Klein GJ, Conacher S, et al. KCNJ2 variant of unknown significance reclassified as long QT syndrome causing ventricular fibrillation. Can J Cardiol. 2011;27:e11-3.

38. Weir RA, Petrie CJ, Murday V, et al. Andersen-Tawil syndrome. Int J Cardiol. 2011;148:e13-5.
39. Sansone V, Tawil R. Management and treatment of AndersenTawil syndrome. Neurotherapeutics. 2007;4:233-7.

40. Haruna Y, Kobori A, Makiyama T, et al. Genotype-phenotype correlations of KCNJ2 mutations in Japanese patients with Andersen-Tawil syndrome. Hum Mutat. 2007;28:208-13.

41. Junker J, Haverkamp W, Schulze-Bahr E, et al. Amiodarone and acetazolamide for the treatment of genetically confirmed severe Andersen syndrome. Neurology. 2002;59:466.

42. Garcia-Touchard A, Somers VK, Kara T, et al. Ventricular ectopy during REM sleep: Implications for nocturnal sudden cardiac death. Nat Clin Pract Cardiovasc Med. 2007;4:284-8.

43. Bökenkamp R, Wilde AA, Schalij MJ, et al. Flecainide for recurrent malignant ventricular arrhythmias in two siblings with Andersen-Tawil syndrome. Heart Rhythm. 2007;4:508-11.

44. Fox DJ, Klein GJ, Hahn A, et al. Reduction of complex ventricular ectopy and improvement in exercise capacity with flecainide therapy in Andersen-Tawil syndrome. Europace. 2008;10:1006-8.

45. Erdogan O, Aksoy A, Turgut N, et al. Oral verapamil effectively suppressed complex ventricular arrhythmias and unmasked $U$ waves in a patient with Andersen-Tawil syndrome. J Electrocardiol. 2008;41:325-8.

46. Haïssaguerre M, Extramiana F, Hocini M, et al. Mapping and ablation of ventricular fibrillation associated with long-QT and Brugada syndromes. Circulation. 2003;108:925-8.

47. Epstein AE, DiMarco JP, Ellenbogen KA, et al., American College of Cardiology Foundation; American Heart Association Task Force on Practice Guidelines; Heart Rhythm Society. 2012 ACCF/AHA/HRS focused update incorporated into the ACCF/AHA/HRS 2008 guidelines for device-based therapy of cardiac rhythm abnormalities: a report of the American College of Cardiology Foundation/American Heart Association Task Force on Practice Guidelines and the Heart Rhythm Society. Circulation. 2013;127:e283-352. 